\title{
Indices of Oxidative Stress in Urine of Patients Undergoing Coronary Artery Bypass Grafting
}

\author{
Wim B. Gerritsen ${ }^{1}$, Leon P. Aarts ${ }^{2}$, Wim J. Morshuis ${ }^{3}$ and Fred J. Haas ${ }^{1}$ \\ ${ }^{1}$ Department of Clinical Chemistry, Sint Antonius Hospital, Nieuwegein, The Netherlands \\ 2 Department of Anaesthesiology, Sint Antonius Hospital, Nieuwegein, The Netherlands \\ ${ }^{3}$ Department of Cardiopulmonary Surgery, Sint Antonius Hospital, Nieuwegein, The Netherlands
}

\begin{abstract}
Summary: Indices of oxidative stress in urine were measured in twenty patients undergoing elective coronary artery bypass grafting. Hypoxanthine, xanthine and uric acid were measured in urine, as markers of ischaemia together with malondialdehyde, which is a marker for lipid peroxidation. To correct for renal dysfunction during coronary artery bypass grafting the creatinine concentration was measured in urine and plasma. The creatinine concentration in plasma increases significantly during surgery, from $84 \pm 23 \mu \mathrm{mol} / \mathrm{l}$ to $133 \pm 52 \mu \mathrm{mol} / \mathrm{l}$, whereas the creatinine concentration in urine decreases significantly, from $8.29 \pm 4.45 \mathrm{mmol} / \mathrm{l}$ to $2.70 \pm 1.01 \mathrm{mmol} / \mathrm{l}$, during reperfusion. For reasons of comparison, the values of the observed measurements in urine are expressed per mol creatinine. The hypoxanthine and xanthine excretions both increase significantly, from $15.0 \pm 7.3$ and $10.9 \pm 5.7$ $\mathrm{mmol} / \mathrm{mol}$ creatinine, respectively, after induction of anaesthesia to a maximum of $33.1 \pm 16.7$ and $17.4 \pm 11.1$ $\mathrm{mmol} / \mathrm{mol}$ creatinine, respectively, during reperfusion. The malondialdehyde excretion increases significantly, from $1.38 \pm 0.80 \mathrm{mmol} / \mathrm{mol}$ creatinine after induction of anaesthesia to a maximum of $3.87 \pm 1.87 \mathrm{mmol} / \mathrm{mol}$ creatinine during reperfusion. The purines and malondialdehyde in urine (expressed as a ratio of creatinine), increase during coronary artery bypass grafting as a consequence of oxygen mediated tissue injury.
\end{abstract}

\section{Introduction}

Oxygen is consumed by cells during the generation of adenosine triphosphate, forming the energetic basis for aerobic cell survival. In the presence of cytochrome oxidase there is a stepwise tetravalent reduction of oxygen to water. However, about $5 \%$ of oxygen is transformed into water by a univalent pathway, in which several reactive oxygen species are produced (1). Reactive oxygen species may be generated under pathological circumstances such as radiation, ischaemia/reperfusion injury and activated white blood cells (2). An increase of reactive oxygen species activity can overwhelm local antioxidant defence and cause damage to biological molecules, especially DNA, lipids and proteins $(3-5)$. Reactive oxygen species play an important role in the pathogenesis of postischaemic myocardial dysfunction (myocardial "stunning", reperfusion injury) after acute myocardial infarction, cardiac surgery and percutaneous transluminal coronary angioplasty $(6-8)$. During and after hypoxia or ischaemia there is, in the heart as well as in other muscles, excessive breakdown of adenosine triphosphate, which causes an efflux of breakdown products like the purine derivatives. These purine derivatives are thought to be good markers for ischaemia, since they are able to pass through the cell membrane and are released into blood. Malondialdehyde is one of the small-molecular-mass compounds

resulting from fragmentation of polyunsaturated fatty acids undergoing reactive oxygen species attack, a fact that qualifies this aldehyde as a marker of lipid peroxidation $(7,17)$.

To identify the potential and actual damage in patients undergoing coronary artery bypass grafting, the following indices in urine are measured: hypoxanthine, xanthine, uric acid and malondialdehyde. The observed increase of the purines and malondialdehyde, confirmed by other investigators $(12,13)$, could be explained by the decrease in renal function that occurs during cardiac surgery (14). Since events as haemolysis, hypotension, hypothermia and non-pulsatile bloodflow during cardiac pulmonary bypass can all contribute to impairment of the renal function, it is not surprising that the purines and malondialdehyde concentration in plasma increase during these conditions.

In this paper we discuss a simple method for the simultaneous determination of the purines and malondialdehyde in urine as a result of oxidative stress. For reasons of comparison, the concentration of hypoxanthine, xanthine, uric acid and malondialdehyde in urine are expressed as ratio of the urinary creatinine and are insensitive for changes in renal function. 


\section{Materials and Methods}

\section{Patients}

Samples were taken from twenty patients undergoing elective first time coronary artery bypass grafting. Seventeen patients were male and three female with mean ages of $69 \pm 13$ years and $73 \pm 4$ years, respectively. Mean time for cardiopulmonary bypass and for aortic crossclamping was $155 \pm 19$, respectively, $111 \pm 16$ minutes.

\section{Samples}

Blood and urinary samples were obtained from patients during and after coronary artery bypass grafting at specific time points, given with their time-intervals $( \pm \mathrm{SD})$ :

1. After induction of anaesthesia; this time is set at: 0 minutes

2. During cardiopulmonary bypass and before aortic crossclamping; $t=105 \pm 11$ minutes

3. After aortic crossclamping until reperfusion; $t=140 \pm 6$ minutes

4. Reperfusion; $\mathrm{t}=251 \pm 16$ minutes

5. Arrival Intensive Care Unit; $t=300 \pm 19$ minutes

6. The first postoperative day $(8.00 \mathrm{a} . \mathrm{m}$.)

Blood samples were taken from the radial artery and collected in tubes containing respectively EDTA $\cdot \mathrm{K}_{2}$ (final concentration $1.6 \mathrm{~g} / 1$ blood) or lithium heparin (final concentration $1.5 \times 10^{3} \mathrm{IU} / 1$ blood). Immediately after withdrawal the lithium heparin blood was placed on ice. Plasma was obtained by centrifugation (lithium heparin blood) at $1500 \mathrm{~g}$ for 10 minutes and the analyses were performed within one hour. Urine specimens were collected during consecutive time points as described above, using containers without any preservative.

\section{Reagents}

HPLC-grade methanol, phosphoric acid $85 \%$ and potassium dihydrogenphosphate (Merck, Darmstadt, Germany), creatinine, tetrabutylammoniumhydroxide, hypoxanthine, xanthine and uric acid (Sigma Chemical Co, St Louis, MO, USA), malondialdehyde tetrabutylammoniumhydroxide (Fluka AG, Buchs, Swiss) were purchased from the respective suppliers.

\section{Analyses}

Albumin, creatinine and uric acid were determined by means of a bromocresol purple dye-binding, a modification of the kinetic Jaffe reaction and the uricase method, respectively, according to the manufacturer's instructions (Dimension $\mathrm{AR}^{\circledR}$ analyzer (Dupont, Wilmington, USA). The haematocrit value was determined on a Coulter STKS ${ }^{\circledR}$ (Coulter Electronics Limited, Luton, England).

Measured values of the different indices in plasma are corrected for haemodilution reported by Beaumont (10).

Corrected value $=$ measured value $\cdot \mathrm{Hct}_{1} \cdot\left(1-\mathrm{Hct}_{2}\right) / \mathrm{Hct}_{2} \cdot\left(1-\mathrm{Hct}_{1}\right)$
In the above equation $\mathrm{Hct}_{1}$ is the initial haematocrit of the first sample (after induction of anaesthesia) and $\mathrm{Hct}_{2}$ is the sample haematocrit. Hypoxanthine, xanthine and malondialdehyde were determined by a slight modification of the HPLC method reported by Lazzarino et al. (9).

\section{HPLC}

Aliquots of a urine specimen, filtered through a sterile acrodisc filter $0.2 \mu \mathrm{m}$ (Gelman Sciences, Ann Arbor, Mi, USA), were analyzed by LKB-HPLC (Pharmacia Biotech AB, Uppsala, Sweden) and separated on a $150 \times 4.6 \mathrm{~mm}$ column of Supelco octadecyl silica (Sulpelco Inc, Bellefonte, PA, USA) using a gradient elution. Absorbance at $272 \mathrm{~nm}$ was monitored and data were collected with a Perkin Elmer 500 series databox by use of Nelson Analytical Chromatography Software, model 2600, Revision 5.0, 1987 (Perkin Elmer Cooperation, Norwalk, USA).

The HPLC method (9) was used to measure indices such as the purines, adenosine triphosphate degradation products and malondialdehyde in one run in plasma. The method was slightly modified. Methanol was omitted in buffer A and the concentration was reduced from $30 \%$ to $20 \%$ in buffer B. The gradient for the chromatographic separation was as follows: 15 minutes at $100 \%$ of buffer A, 15 minutes up to $100 \%$ of buffer B and hold for additional 6 minutes. The initial conditions were restored after 9 minutes of washing with buffer A. Instead of plasma we used urine to determine the purines and malondialdehyde. Technical problems during sample handling when plasma is used are thus prevented. A good reproducibility of the method was found with an intra-run coefficient of variation of $1.1 \%$ for hypoxanthine, xanthine and uric acid to $3.1 \%$ for malondialdehyde and a detection limit of $0.2 \mu \mathrm{mol} / \mathrm{l}$, for all indices. Thereby, the measurement of hypoxanthine, xanthine, uric acid and malondialdehyde in urine shows an excellent resolution and peaks are easy to identify. However urine samples stored by $-70^{\circ} \mathrm{C}$ are not stable and the values decrease in time (result not shown). To minimize this storage effect all analyses where performed within one hour.

\section{Statistics}

Comparisons between groups were made by repeated measures using ANOVA and the Student's t-test. $\mathrm{P}<0.05$ was considered to indicate statistical significance.

\section{Results}

Measurements in plasma

\section{Haematocrit}

After induction of anaesthesia the mean haematocrit value of the patients was $0.36 \pm 0.03 \mathrm{l} / \mathrm{l}$. During reperfusion the haematocrit decreased significantly to 0.23 $\pm 0.02 \mathrm{l} / \mathrm{l}$. The first postoperative day, the haematocrit

Tab. 1 Correction of indices in plasma according to the method of Beaumont

\begin{tabular}{|c|c|c|c|c|}
\hline \multirow[t]{2}{*}{ Time points } & \multirow{2}{*}{$\begin{array}{l}\text { Haematocrit* } \\
1 / 1\end{array}$} & \multicolumn{3}{|c|}{ Corrected mean of the indices } \\
\hline & & $\begin{array}{l}\text { Albumin } \\
\mathrm{g} / 1\end{array}$ & $\begin{array}{l}\text { Creatinine* } \\
\mu \mathrm{mol} / 1\end{array}$ & $\begin{array}{l}\text { Uric acid* } \\
\mu \mathrm{mol} / 1\end{array}$ \\
\hline After induction of anaesthesia & $0.362 \pm 0.028$ & $31.0 \pm 2.3$ & $84.2 \pm 23.3$ & $280 \pm 60$ \\
\hline During cardiopulmonary bypass and before aortic crossclamping & $0.353 \pm 0.037$ & $30.4 \pm 3.3$ & $87.6 \pm 23.1$ & $300 \pm 80$ \\
\hline After aortic crossclamping until reperfusion & $0.233 \pm 0.050$ & $32.3 \pm 5.1$ & $139.5 \pm 43.8$ & $530 \pm 160$ \\
\hline Reperfusion & $0.234 \pm 0.024$ & $33.7 \pm 5.6$ & $133.8 \pm 52.3$ & $510 \pm 120$ \\
\hline Arrival Intensive Care Unit & $0.276 \pm 0.035$ & $32.6 \pm 5.9$ & $116.0 \pm 43.7$ & $400 \pm 120$ \\
\hline The first postoperative day & $0.327 \pm 0.017$ & $31.8 \pm 3.7$ & $119.9 \pm 45.7$ & $380 \pm 100$ \\
\hline
\end{tabular}

* $\mathrm{p}<0.05$ by repeated measured ANOVA 
value was restored to $0.33 \pm 0.02 \mathrm{1} / 1$ without transfusion of erythrocyte concentrate (tab. 1).

\section{Albumin}

After correction for haemodilution, the mean value of albumin was $31.5 \pm 2.5 \mathrm{~g} / 1$, which remains constant during surgery, indicating that the correction as described by Beaumont (10) is an acceptable correction (tab. 1).

\section{Creatinine}

During coronary artery bypass grafting the mean creatinine concentration in plasma increased significantly, from $84 \pm 23 \mu \mathrm{mol} / 1$ after induction of anaesthesia to $134 \pm 52 \mu \mathrm{mol} / \mathrm{l}$ during reperfusion. At the first postoperative day the creatinine concentration decreased to 120 $\pm 46 \mu \mathrm{mol} / \mathrm{l}$. During coronary artery bypass grafting there is an accumulation of creatinine due to a decreased renal function (fig. 1a, tab. 1).

\section{Uric acid}

There is a strong increase in the concentration of uric acid during coronary artery bypass grafting. The mean concentration of uric acid was $0.28 \pm 0.06 \mu \mathrm{mol} / 1$ after
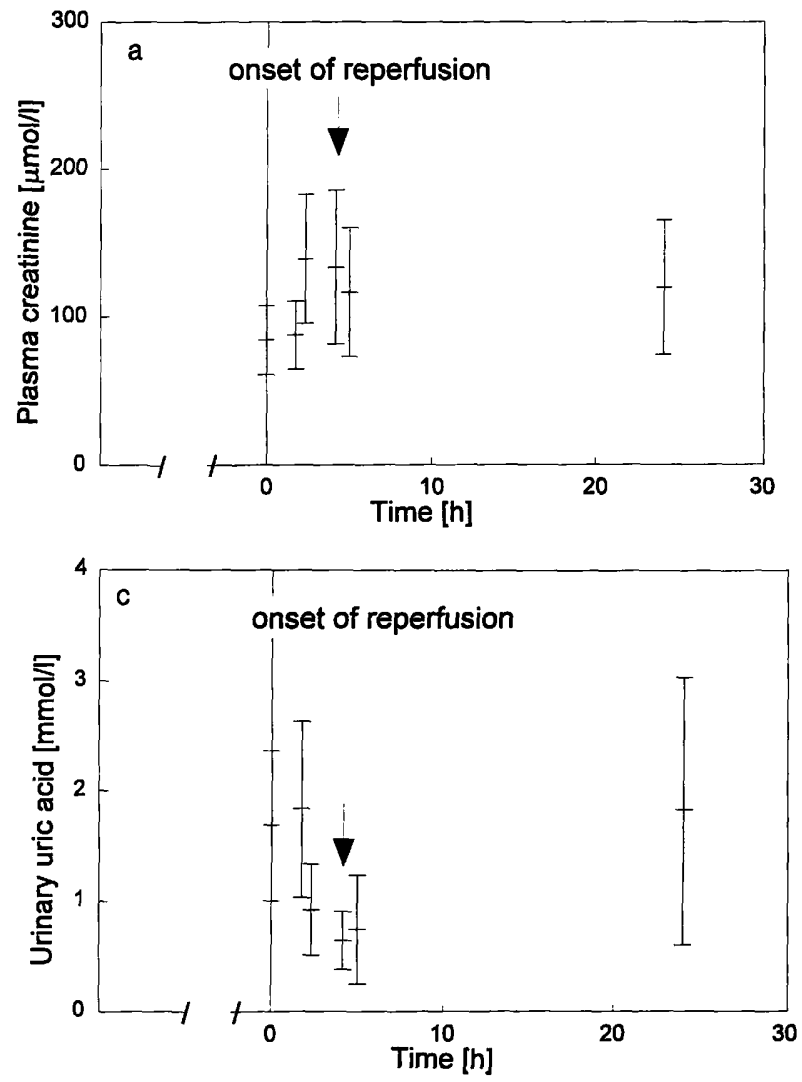

Fig. 1 Creatinine in plasma and urine during coronary artery bypass grafting surgery at specific time points.

(a) Values are mean \pm SD of creatinine in plasma of twenty subjects $(\mathrm{P}<0.05)$.

(b) Values are mean \pm SD of creatinine in urine of twenty subjects $(P<0.05)$. induction of anaesthesia and increased significantly to $0.51 \pm 0.12 \mu \mathrm{mol} / 1$ during reperfusion. At the first postoperative day the uric acid concentration decreased again to $0.38 \pm 0.10 \mu \mathrm{mol} / 1$ (tab. 1). However, when the concentration of uric acid is expressed as a ratio of creatinine the concentrations of uric acid were almost identical before, during, and after coronary artery bypass grafting with a mean value of $0.29 \pm 0.07 \mu \mathrm{mol} / 1$.

\section{Measurements in urine}

\section{Creatinine}

During coronary artery bypass grafting the mean creatinine concentration decreased significantly from 8.29 $\pm 4.45 \mathrm{mmol} / \mathrm{l}$ after induction of anaesthesia to 2.70 $\pm 1.01 \mathrm{mmol} / 1$ during reperfusion. The first postoperative day the mean creatinine concentration was increased significantly, to a value of $6.90 \pm 3.65 \mathrm{mmol} / \mathrm{l}$ (fig. $\mathrm{lb}$, tab. 2).

\section{Uric acid}

The profile of the uric acid concentration is identical to the profile of the creatinine concentration during coronary artery bypass grafting (fig. 1c, tab. 2); the concentration
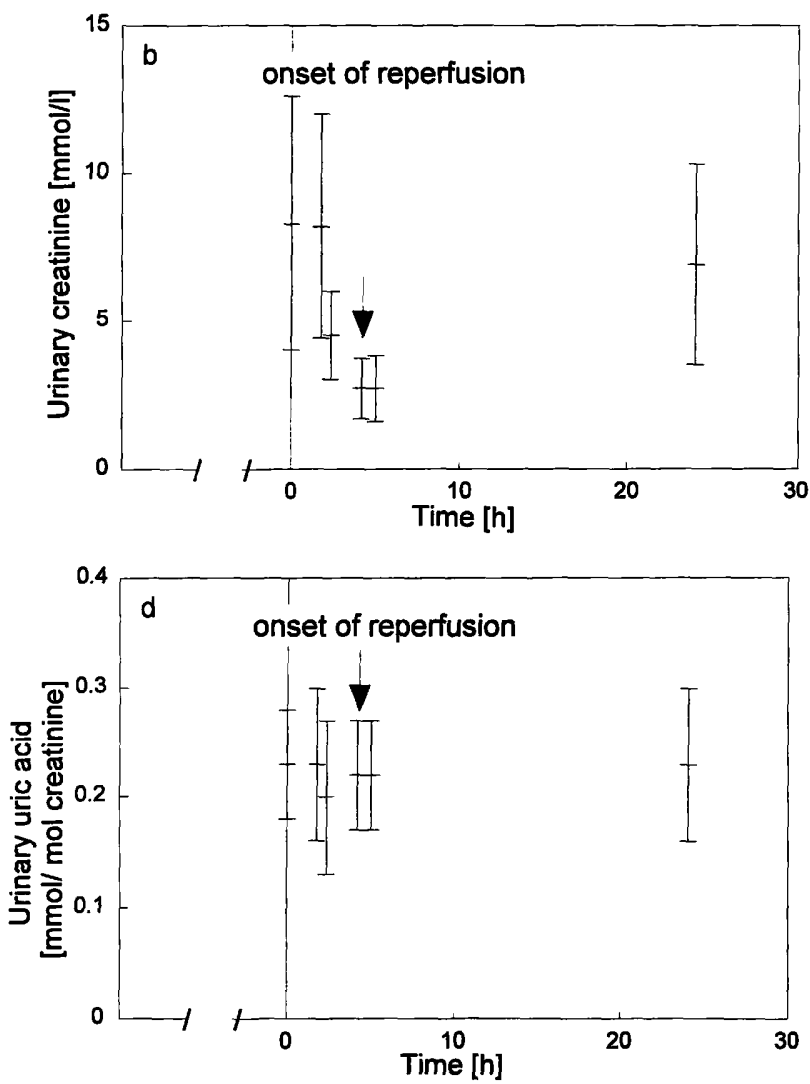

Uric acid in urine during coronary artery bypass grafting surgery at specific time points.

(c) Values are mean \pm SD of uric acid in urine of twenty subjects $(P<0.05)$.

(d) Values are mean \pm SD of uric acid in urine of twenty subjects expressed as ratio of urinary creatinine $(P>0.80)$. 
Tab. 2 Indices measured in urine

\begin{tabular}{|c|c|c|c|c|c|}
\hline Time points & $\begin{array}{l}\text { Creatinine* } \\
\mathrm{mmol} / \mathrm{l}\end{array}$ & $\begin{array}{l}\text { Uric acid* } \\
\text { mmol/1 }\end{array}$ & $\begin{array}{l}\text { Hypoxanthine }{ }^{* 1} \\
\mu \mathrm{mol} / 1\end{array}$ & $\begin{array}{l}\text { Xanthine* } \\
\mu \mathrm{mol} / 1\end{array}$ & $\begin{array}{l}\text { Malondi- } \\
\text { aldehyde*2 } \\
\mu \mathrm{mol} / 1\end{array}$ \\
\hline After induction of anaesthesia & $8.3 \pm 4.3$ & $1.68 \pm 0.68$ & $116.8 \pm 63.4$ & $81.7 \pm 35.1$ & $11.2 \pm 8.3$ \\
\hline $\begin{array}{l}\text { During cardiopulmonary bypass and before aortic } \\
\text { crossclamping }\end{array}$ & $8.2 \pm 3.8$ & $1.83 \pm 0.80$ & $111.1 \pm 39.3$ & $80.2 \pm 26.3$ & $8.4 \pm 5.7$ \\
\hline After aortic crossclamping until reperfusion & $4.5 \pm 1.5$ & $0.92 \pm 0.41$ & $75.0 \pm 31.3$ & $52.0 \pm 25.0$ & $7.4 \pm 2.5$ \\
\hline Reperfusion & $2.7 \pm 1.0$ & $0.64 \pm 0.26$ & $84.4 \pm 44.9$ & $41.7 \pm 22.8$ & $9.8 \pm 5.4$ \\
\hline Arrival Intensive Care Unit & $2.7 \pm 1.1$ & $0.74 \pm 0.49$ & $73.0 \pm 44.7$ & $49.4 \pm 38.8$ & $5.2 \pm 4.1$ \\
\hline The first postoperative day & $6.9 \pm 3.4$ & $1.81 \pm 1.22$ & $115.9 \pm 63.2$ & $70.5 \pm 26.8$ & $4.8 \pm 2.8$ \\
\hline
\end{tabular}

${ }^{*} \mathrm{p}<0.05,{ }^{* 1}: \mathrm{p}<0.35 ;{ }^{* 2}: \mathrm{p}<0.20$ by repeated measures using ANOVA

decreases strongly during reperfusion. When the uric acid values are expressed as ratio of the urinary creatinine, there is no significant difference $(p>0.80)$ in time (fig. 1d).

\section{Hypoxanthine}

The mean hypoxanthine excretion expressed as ratio of the urinary creatinine increased significantly, from 15.0 $\pm 7.3 \mathrm{mmol} / \mathrm{mol}$ creatinine after induction of anaesthesia to $33.1 \pm 16.7 \mathrm{mmol} / \mathrm{mol}$ creatinine during reperfusion. The first postoperative day the mean hypoxanthine excretion decreased to the starting concentration of 19.1 $\pm 11.5 \mathrm{mmol} / \mathrm{mol} \mathrm{creatinine} \mathrm{(fig.} 2 \mathrm{a}$ ).

\section{Xanthine}

The mean xanthine excretion, expressed as ratio of the urinary creatinine, increased significantly, from 10.9 $\pm 5.7 \mathrm{mmol} / \mathrm{mol}$ creatinine after induction of anaesthesia, to a maximum of $18.9 \pm 9.0 \mathrm{mmol} / \mathrm{mol}$ creatinine at arrival on the Intensive Care Unit. At the first postoperative day, the mean xanthine excretion was lowered to $12.9 \pm 9.9 \mathrm{mmol} / \mathrm{mol}$ creatinine (fig. $2 \mathrm{~b}$ ).

\section{Malondialdehyde}

The mean malondialdehyde excretion, expressed as ratio of the urinary creatinine, increased significantly, from $1.38 \pm 0.80 \mathrm{mmol} / \mathrm{mol}$ creatinine after induction of anaesthesia, to a maximum of $3.87 \pm 1.87 \mathrm{mmol} / \mathrm{mol}$ creatinine during reperfusion. At the first postoperative day the mean value decreased significantly, to $0.87 \pm 0.40$ $\mathrm{mmol} / \mathrm{mol}$ creatinine (fig. $2 \mathrm{c}$ ).

\section{Discussion}

We evaluated indices of ischaemia and oxidative stress in twenty patients undergoing elective first time coronary artery bypass grafting. Although the myocardium is well protected by cardioplegia during coronary artery bypass grafting, there is still ischaemia resulting in breakdown of adenosine triphosphate to hypoxanthine and other degradation products (11). Activated proteases, induced by $\mathrm{Ca}^{2+}$, convert the enzyme xanthine de- hydrogenase into xanthine oxidase. During reperfusion, hypoxanthine will be converted by xanthine oxidase to xanthine and uric acid (19). Reactive oxygen species are generated during the xanthine oxidoreductase route and cause tissue injury $(8,15,16)$.

The plasma concentrations of albumin, creatinine and uric acid during coronary artery bypass grafting were corrected for haemodilution (10). As expected, the corrected concentration of albumin remains constant during surgery indicating that the correction used is valid for patients with haemodilution. The concentration of creatinine and uric acid increases strongly during coronary artery bypass grafting. Other studies $(12,13)$ reported increased concentrations of purines and malondialdehyde in plasma during coronary artery bypass grafting. The observed increase of creatinine and uric acid and the reported increase of hypoxanthine, xanthine and malondialdehyde in plasma could both be explained by renal dysfunction that occurs during cardiac surgery (14). To determine the individual influence of hypotension and non-pulsatile bloodflow during cardiac pulmonary bypass, changes in creatinine in plasma as well in urine are functionally good markers (14). Therefore all urinary indices are expressed as a ratio of the creatinine concentration in urine and are in this way insensitive to alterations in renal function. Thereby, we assume that clearance of small molecules such as creatinine and malondialdehyde are comparable because these are only filtered by the kidneys (passive excretion), whereas for uric acid (and probably the purines) filtration, tubular reabsorption and tubular secretion are evident. In order to correct and normalize all the indices in the same manner, we express the indices as a ratio of the urinary creatinine. The excretion of hypoxanthine, expressed as ratio of creatinine, increases significantly, from $15.0 \pm 7.3 \mathrm{mmol} /$ mol creatinine after induction of anaesthesia to a maximum of $33.1 \pm 16.7 \mathrm{mmol} / \mathrm{mol}$ creatinine during reperfusion. Hypoxanthine was formed during the ischaemic period of the myocardium (20). During reperfusion, hypoxanthine will be converted by xanthine oxidase to xanthine and uric acid. A part of the produced hypoxanthine will be cleared by the kidneys in a time dependent process. The excretion of xanthine increases signifi- 
cantly, from $10.9 \pm 5.7 \mathrm{mmol} / \mathrm{mol}$ creatinine after induction of anaesthesia to a maximum of $18.9 \pm 9.0$ $\mathrm{mmol} / \mathrm{mol}$ creatinine at arrival on the Intensive Care Unit. These results were as expected because the conversion of hypoxanthine into xanthine took place after reperfusion. The first postoperative day the concentrations of both indices decrease significantly to the starting excretion of $19.1 \pm 11.5 \mathrm{mmol} / \mathrm{mol}$ creatinine for hypoxanthine and $12.9 \pm 9.9 \mathrm{mmol} / \mathrm{mol}$ creatinine for xanthine. After correction of the ischaemia of the myocardium by bypassing the coronary arteries, the formation
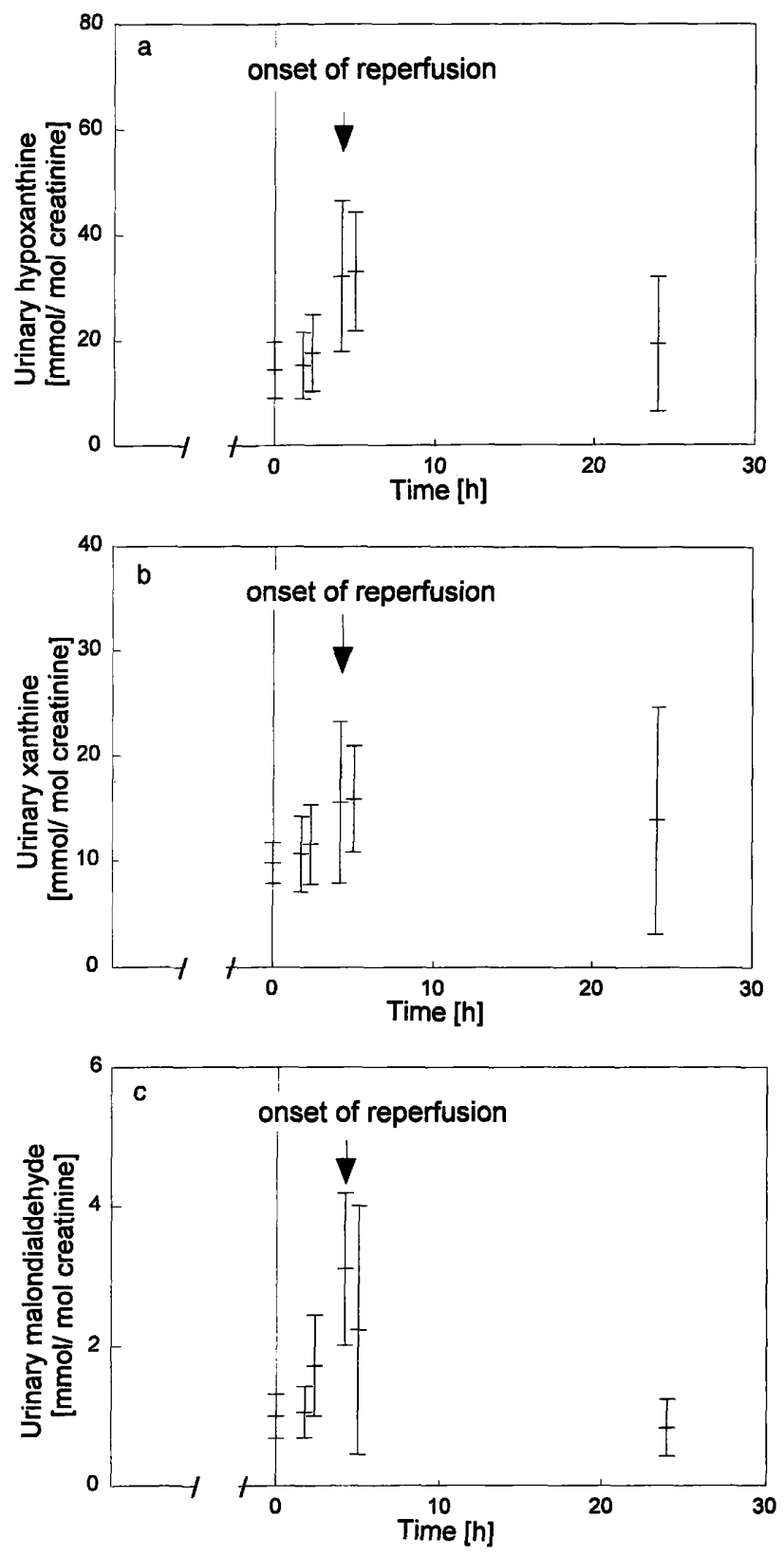

Fig. 2 Purines and malondialdehyde in urine during coronary artery bypass grafting surgery at specific time points.

(a) Values are mean $\pm \mathrm{SD}$ of hypxanthine in urine of twenty subjects expressed as ratio of urinary creatinine $(P<0.05)$.

(b) Values are mean \pm SD of xanthine in urine of twenty subjects expressed as ratio of urinary creatinine $(P<0.16)$.

(c) Values are mean \pm SD of malondialdehyde in urine of twenty subjects expressed as ratio of urinary creatinine $(P<0.05)$. of adenosine triphosphate degradation products ceases. The purines will be cleared by the kidney and the excretion will normalize in time.

On the other hand, the excretion of uric acid decreases strongly during reperfusion. Presenting the results of uric acid as ratio of the creatinine in urine, there is no significant difference between the time points for uric acid (fig. 1d). The expected increase of the concentration of uric acid, which occurs as a result of the xanthine oxidoreductase route due to ischaemia/reperfusion, did not occur. Probably the method used is not sensitive enough to discriminate between these small increases. Moreover uric acid probably acts as an anti-oxidant, so that the free radicals produced during ischaemia will be trapped by uric acid whereby uric acid is converted into allantoine (18).

In patients with heart failure the excretion of malondialdehyde was actually higher than that measured postoperatively as was reported earlier (12). We compared the excretion of malondialdehyde of twenty healthy volunteers, mean excretion $\pm 1 \mathrm{SD}: 0.20 \pm 0.05 \mathrm{mmol} / \mathrm{mol}$ creatinine, with the excretion of malondialdehyde from the patients investigated, $1.38 \pm 0.80 \mathrm{mmol} / \mathrm{mol}$ creatinine, undergoing elective first time coronary artery bypass grafting. There was a significant difference in the excretion of malondialdehyde between the two groups $(p<0.05)$, indicating that there was a difference in the ischaemic status. During reperfusion the excretion of malondialdehyde expressed as a ratio of creatinine reaches a maximum of $3.87 \pm 1.87 \mathrm{mmol} / \mathrm{mol}$ creatine. At the first postoperative day the excretion of malondialdehyde decreases significantly, to $0.87 \pm 0.40 \mathrm{mmol} /$ mol creatinine, indicating less ischaemic myocardium and that the intervention was successful. For logistic reasons we stopped sampling after the first post-operative day. No data are available about the concentration of malondialdehyde over time.

In general, the excretion of the purines, hypoxanthine, xanthine and uric acid, measured during coronary artery bypass grafting, is still increased despite the correction for creatinine as an indicator for renal function. The increase of the concentrations of purines and malondialdehyde in plasma is probably caused by a reduced clearance of these products by the kidneys instead of by ischaemic reperfusion injury (13). Comparing the excretions of purines and malondialdehyde, corrected for changes in renal function in time, gives a good impression of the oxidative tissue injury by reactive oxygen species.

\section{Acknowledgements}

We thank Kai Liang, Sigrid Jansen, Hans van Dam and Fachreddin Abarchan for their technical assistance and Douwe van Loon for editorial help. 


\section{References}

1. Aarts L. Anaesthesia and oxidative stress [thesis]. Amsterdam Vrije Universiteit, 1995: 1-10.

2. Princemail J, Deby-Dupont G. Biochemical alterations in ischemia and reperfusion. In: Vincent JL, editor. Yearbook of intensive care and emergency medicine. Berlin - New York: Springer Verlag 1992:104-14.

3. Miller RA, Britigan BE. The formation and biologic significance of phagocyte-derived oxidants. J Invest Med 1995; 43:39-49.

4. Reilly PM, Schiller HJ, Bulkley. Pharmacologic approach to tissue injury mediated by free radicals and other reactive oxygen metabolites. Am J Surg 1991; 161:481-503.

5. Cerutti PA. Oxy-radicals and cancer. Lancet 1994; 344:862-3.

6. Ames BN, Shigensaga MK, Hagen TM. Oxidants, antioxidants, and the degenerative diseases of aging. Proc Natl Acad Sci USA 1993; 90:7915-22.

7. Toivonen HJ, Ahotupa M. Free radical reaction products and antioxidant capacity in arterial plasma during coronary artery bypass grafting. J Thorac Cardiovasc Surg 1994; 108:140-7.

8. Prasad K, Kalra J, Bhawaradwaj B, Chaudhary AK. Increased oxygen free radical activity in patients on cardiopulmonary bypass undergoing aortocoronary bypass surgery. Am Heart J 1992; 123:37-45.

9. Lazzarino G, Di Pierro D, Tavazzi B, Cerroni L, Giardina B. Simultaneous separation of malondialdehyde and adenine nucleotide derivatives from biological samples by ion-pairing high performance liquid chromatography. Anal Biochem $1991 ; 197: 191-6$.

10. Beaumont van W. Evaluation of haemoconcentration from haematocrit measurements. J Appl Physiol 1972; 32:712-3.

11. Bical O, Gerhardt MF, Paumier D, Gaillard D, Comas J, Landais $\mathrm{P}$, et al. Comparison of different types of cardioplegia and reperfusion on myocardial metabolism and free radical activity. Circulation 1991; 81-III:373-9.

12. Diaz-Velez CR, Garcia-Castiñeras S, Mendoza-Ramos E, Hernandez-Lopez E. Increased malondialdehyde in peripheral blood of patients with congestive heart failure. Am Heart J 1996 ; 131:146-52.
13. Lazzarino G, Raatikainen $P$, Nuutinen $M$, Nissinen $M$, Tavazzi B, Di Pierro D, et al. Myocardial release of malondialdehyde and purine compounds during coronary bypass surgery. Circulation 1994; 90:291-7.

14. Koning HM, Koning AJ, Defauw JJAM. Optimal reperfusion during extra-corporeal circulation. Scand J Thor Cardiovasc Surg 1987; 21:207-13.

15. De Scheerder IK, Van de Kraay AM, Lamers JMJ, Koster JF, De Jong JW, Serruys PW., Myocardial malondialdehyde and uric acid release after short-lasting coronary occlusions during coronary angioplasty: potential mechanism for free radical generation. Am J Card 1991; 68:392-5.

16. Bast A, Haenen GR, Doelman CJ. Oxidants and anti-oxidants: state of the art. Am J Med 1991; 91(3C):2S-13S.

17. Esterbauer H, Cheeseman KH. Determination of aldehydic lipid peroxidation products: malonaldehyde and 4-hydroxynonenal. Meth Enzymol 1990; 186;407-21.

18. Kock R, Delvoux B, Sigmund, Greiling H. A comparative study of the concentrations of hypoxanthine, xanthine, uric acid and allantoin in the pheripheral blood of normals and patients with acute myocardial infarction and other ischaemic diseases. Eur J Clin Chem Clin Biochem 1994; 32:837-42.

19. Bulkley GB. Reactive oxygen metabolites and reperfusion injury: aberrant triggering of reticuloendothelial function. Lancet $1994 ; 344: 934-6$.

20. Jaarsveld van H, Barnard HC, Barnard SP, Maartens JJ, Potgieter GM. Purine and oxypurine production in mitochondria of ischemic and reperfused myocardium. Enzyme 1989; $42: 136-44$

Received April 10/July 15, 1997

Corresponding author: Fred J Haas, Department of Clinical Chemistry, Sint Antonius Hospital, P. O. Box 2500, NL-3430 EM Nieuwegein, The Netherlands

Fax: +31.30.6092528, e-mail: F.Haas@kcl-azn.demon.nl 